\section{Feeding China's growing needs for grain}

State farms, modelled on those successful in northeastern China, could meet burgeoning grain demand in the rest of the country over the next 20 years.

China already grows more than $95 \%$ of its own grain, providing food security to $22 \%$ of the world's population even though it has only $9 \%$ of the world's cultivated farmland (see go.nature.com/zhKwtg; go.nature. $\mathrm{com} / \mathrm{fA} 9 \mathrm{TXv}$ ). But we estimate that a total of almost 100 billion kilograms more grain will be needed by 2030 (X. Liu and G. Wang Rhizosphere and Yield Physiology of Crops 8-9, Science Press, 2010; in Chinese).

This rising demand will call for a $1 \%$ annual increase in total grain production from the same 120 million hectares of cultivated farmland currently available. The only way to step up crop yields is by intensifying production.

State farms in the 'bread basket' of northeastern China have almost doubled their crop yields per unit area compared with the region's local farms.

Their average increase in yield during the past 15 years for maize (corn), soya bean and rice, for example, has been $32.6 \%, 27.8 \%$ and $19.9 \%$, respectively. Owing to their greater land area, had local farms adopted similar technology and operational management, their annual grain yield would have been boosted by $82.3 \%$, $66.1 \%$ and $32.2 \%$, respectively, for the same crops (Heilongjiang Statistics Yearbook 2008; in Chinese). That is potentially 1.6 times more grain from the same farmland.

Extending this model to other regions in China will bring challenges, including declining water levels, a surplus of farm labour and the need to develop new services to accommodate changes in agricultural structure.

Nonetheless, there is an opportunity here for China to solve its future grain demand through technological solutions and by rationalizing institutional and organizational irregularities (D. W. Bromley Science 328, 169; 2010). Meanwhile, it could reassess its policies for land management, urbanization processes and subsidies.

\section{Xiaobing Liu, Xingyi Zhang}

Northeast Institute of Geography and Agroecology, Chinese Academy of Sciences, Harbin 150081, China e-mail: liuxb@neigae.ac.cn

Stephen J. Herbert Center for Agriculture, University of Massachusetts, Amherst, Massachusetts 01003, USA

\section{Changing climate threatens tropical rainforests too}

The trailing edges of retreating tropical forests are under-studied. And they are under even greater pressure than temperate forests from the direct and synergistic effects of climate change (Opinion, Nature 464, 1271; 2010).

In rainforest remnants of equatorial Singapore, understorey ecosystems could be becoming dangerously dry. During a neardrought in February and March this year, the water table sank and some forest streams temporarily disappeared from the country's two nature reserves.

Rainfall is likely to become more seasonal under changing global climate patterns such as El Niño (S. Yeh et al. Nature 461, 511-514; 2009). This is expected to make a larger contribution than warming in the shift towards arid conditions in the perhumid tropics, which normally have plentiful rain throughout the year. How tropical rainforest plants will respond to the new conditions is unclear, because field measurements are rare. Unlike temperate forests, no dramatic diebacks have been documented and we have fewer historical clues.

There may also be insidious changes under way in the community composition of rainforests in tropical Asia. In Singapore, beach-type forest species such as Calophyllum ferrugineum and Syzygium grande (sea apple) are becoming increasingly dominant among seedlings in the inland forest understorey. Mass fruiting of dipterocarps, the dominant plant family in tropical Asian rainforests, has been triggered more frequently as El Niño events have increased. With less time to accumulate resources, parent trees may be forced to produce smaller seeds that have less chance of survival.

Synergistic interactions with other conservation threats could also be a bigger problem in tropical forests, which have many more species and more complex mutualisms than temperate forests.

Kwek Yan Chong, Chow Khoon Yeo, Alex Thiam Koon Yee Plant Systematics Laboratory, Department of Biological Sciences, National University of Singapore, 14 Science Drive 4, Singapore 117543 e-mail:kwek@nus.edu.sg

\section{European Commission responds to chemical testing story}

As the European Commission spokesperson for Research, Innovation and Science, I wish to correct your misleadingly entitled News report 'Crucial data on REACH not disclosed' (Nature 464, 1116-1117; 2010).

The commission refutes as factually and contextually incorrect any suggestion that "crucial data" were not disclosed. As toxicologist Thomas Hartung's case is ongoing, the commission cannot comment further on his claims, or on the proceedings to ascertain potential previous instances of professional misconduct.

However, the facts are as follows. In the longer term, REACH (Registration,
Evaluation, Authorisation and Restriction of Chemicals) should reduce animal testing. But an assessment completed by the commission's Joint Research Centre (JRC) in September 2006 suggested that a minimum of 8 million to 9 million additional animals would be required in the short- to medium-term.

A summary of this assessment was published on the website of the JRC Institute for Health and Consumer Protection on 27 November 2006 (and not in January 2007).

Contrary to your report, the assessment was therefore continuously publicly available throughout the period when the European Parliament approved REACH on 13 December 2006 and when national ministers in the Council of the European Union approved it definitively in summer 2007. A slightly amended version of the assessment containing a disclaimer was published on 16 December 2006 (see go.nature.com/ZMguvW).

This document has been superseded by a more recent assessment by the European Chemicals Agency, which confirms the approximate figure of 8 million to 9 million animals. The commission is a stakeholder in the agency (see go.nature.com/ uw79bC).

The commission emphasizes that the work of its JRC is collaborative with national and international partners, and that its assessments are never the view of one individual. In performing its mission of scientific and technical support, the JRC follows stringent qualitymanagement and peer-review procedures.

Mark English European Commission, 1049 Brussels, Belgium e-mail: mark.english@ec.europa.eu

\footnotetext{
Contributions may be submitted to correspondence@nature.com; see go.nature.com/cMCHno.

Readers can now comment online on anything published in Nature: www.nature.com/nature.
} 